\title{
Alteraciones radiológicas y movilidad de la columna vertebral observadas en pilotos de combate españoles
}

\author{
Carbayo Herencia JA. ${ }^{1}$, Velasco Díaz C. ${ }^{2}$, Sánchez Nievas G. ${ }^{3}$, Abad Ortiz L. . , Caldevilla Bernardo D. ${ }^{4}$
}

Sanid. mil. 2011; 67 (4): 361-366; ISSN: 1887-8571

\begin{abstract}
RESUMEN:
Antecedentes y Objetivos: El dolor de espalda es padecido por la mayoría de pilotos de combate. Los objetivos del presente estudio han sido valorar en esta población si aparecen lesiones precoces de la columna vertebral y la movilidad de ésta. Material y Métodos: Participaron 30 pilotos de combate y 16 militares como grupo de comparación (GC), no pilotos, destinados en la Base Aérea de Albacete, España. A todos se les practicó 6 radiografías (dos en la columna cervical, dos en la columna dorsal y dos en la región lumbosacra). Se calculó la razón de prevalencias (RP) de las lesiones valoradas entre los dos grupos, considerando significativo un valor de $\mathrm{P} \leq 0,05$. Resultados: Tanto la edad media de los pilotos $(31,9$ años $[\mathrm{DE}=5,9])$ como la del $\mathrm{GC}(32,3$ años $[\mathrm{DE}=3,4])$, así como su índice de masa corporal $\left(24,4 \mathrm{~kg} / \mathrm{m}^{2}\right.$ en los pilotos frente a 24,4 [DE=1,9] del GC) fueron similares. Encontramos diferencias significativas entre los dos grupos en las siguientes variables: uncoartrosis $(\mathrm{RP}=2,4 ; \mathrm{P}=0,02)$, artrosis interapofisarias $(\mathrm{RP}=2,82 ; \mathrm{P}=0,02)$, osteofitos $(\mathrm{RP}=4,26 ; \mathrm{P}=0,007)$, pérdida de altura del cuerpo vertebral $(\mathrm{RP}=11,29 ; \mathrm{P}=0,008)$ en la región cervical, e irregularidades subcondrales $(\mathrm{RP}=2,82 ; \mathrm{P}=0,02)$ en la región dorsal. La movilidad de la espalda (valorada por flexiones en las distintas regiones, extensiones, rotaciones y lateralizaciones) fue menor en el piloto de combate. Conclusiones: Los pilotos de combate de nuestro estudio presentan una mayor rigidez en la columna vertebral, asi como alteraciones radiológicas precoces, principalmente en la región cervical, comparados con un GC similar.
\end{abstract}

PALABRAS CLAVE: Piloto de combate, Dolor de espalda, Dolor cervical, Lumbalgia, Medicina preventiva.

\section{Radiological alterations and spine mobility in Spanish fighter pilots}

SUMMARY:

Background and Objectives: Back pain is suffered by most fighter pilots. The objectives of this study were evaluated in this population if there are early lesions of the spine and their mobility. Material and Methods: The participants were 30 fighter pilots and 16 soldiers as a comparison group (CG), no fighter pilots, stationed in the Air Base in Albacete, Spain. All 6 underwent X-rays (two in the cervical spine, two in the thoracic spine and two in the lumbosacral region). We calculated the prevalence ratio (PR) of the lesions assessed between the two groups was considered significant a $\mathrm{P}$ value $\leq 0.05$. Results: Both the average age of fighter pilots $(31.9$ years [SD $=5.9])$ as the $\mathrm{CG}$ (32.3 years $[\mathrm{SD}=3.4])$ and body mass index $(24,4 \mathrm{~kg} / \mathrm{m} 2$ in fighter pilots compared to 24.4 [SD $=1.9]$ of the $\mathrm{CG})$ were similar. We found significant differences between the two groups in the following variables: uncoartrosis $(\mathrm{PR}=2.4, \mathrm{P}=0.02)$, apophyseal osteoarthritis ( $\mathrm{PR}$ $=2.82, \mathrm{P}=0.02)$, osteophytes $(\mathrm{PR}=4.26 \mathrm{P}=0.007)$, loss of vertebral body height $(\mathrm{PR}=11.29, \mathrm{P}=0.008)$ in the cervical region, subchondral irregularity $(\mathrm{PR}=2.82, \mathrm{P}=0.02)$ in the dorsal region. The mobility of the back (as measured by bending in different areas, extensions, rotations and lateralized) was lower in the fighter pilot. Conclusions: The fighter pilots of our study have a higher stiffness in the spine, as well as early radiological changes, mainly in the cervical region, compared with a similar CG.

KEY WORDS: Fighter pilot, Back pain, Neck pain, Low back pain, Preventive medicine.

\section{INTRODUCCIÓN}

El dolor de espalda, especialmente el localizado en la región cervical, es un síntoma frecuentemente manifestado por los pilotos de combate, ampliamente conocido ${ }^{1}$, también registrado por nosotros en un estudio previo, en el cual el $85 \%$ de los pilotos valorados refirieron haber padecido en alguna ocasión dolor de espalda ${ }^{2}$. Esta percepción, más la exposición frecuente a altas aceleraciones como característica fundamental de su profesión, hace que nos hayamos

Tcol. Médico. Médico de Vuelo. Base Aérea de Albacete. Albacete. España.

2 Tcol Médico. Centro de Instrucción de Medicina Aeroespacial (CIMA). Madrid. España.

${ }^{3}$ Servicio de Reumatología. Hospital General Universitario de Albacete. Albacete. España.

${ }^{4}$ Servicio de Radiología. Hospital General Universitario de Albacete. Albacete. España.

Dirección para correspondencia: Julio A. Carbayo Herencia. Sección de Sanidad. Base Aérea de Albacete. Carretera de Murcia s/n. 02071 Albacete. Teléfono: 967556256 y 967556276. jacarbayoh@telefonica.net

Recibido: 16 de septiembre de 2010

Aceptado: 17 de febrero de 2011 propuesto como objetivos principales del presente estudio comprobar si aparecen alteraciones precoces en la columna vertebral del piloto detectadas por radiología simple y de ser así, si éstas están relacionadas con el dolor de espalda manifestado, así como valorar la movilidad de la columna vertebral.

\section{MATERIAL Y MÉTODOS}

El diseño corresponde a un estudio no experimental, transversal en el que la variable independiente es el hecho de volar, presente en todos los pilotos, analítico, con un grupo de comparación seleccionado por apareamiento, de similar edad y trayectoria profesional, excepto el hecho de volar.

Intervinieron en el presente estudio un total de 30 pilotos de combate destinados en la Base Aérea de Albacete y 17 participantes pertenecientes a un grupo de comparación, militares profesionales no pilotos. Un participante del grupo control fue excluido por pre- 
sentar la enfermedad de Forrestier y Rotés-Querol, de modo que los participantes definitivos de este grupo fueron 16. Todos eran varones, al igual que los pilotos, los cuales además cumplimentaron un cuestionario que incluía las horas de vuelo efectuadas y el dolor de espalda percibido, así como sus características. Dicho cuestionario ya fue expuesto en una publicación previa ${ }^{2}$. Para facilitar su comprensión se reproduce parte de ella como tabla 1.

A todos los participantes (pilotos y grupo control) se les realizó 6 radiografías, correspondiendo dos a la región cervical (anteroposterior y lateral), dos a la región dorsal (anteroposterior y lateral) y las dos restantes (igualmente anteroposterior y lateral) a la región lumbosacra.

Para mantener la mínima variabilidad, todas las radiografías (excepto un caso) se realizaron en el mismo centro, con la misma metodología y bajo la dirección de uno de los autores, especialista en Radiología.

En la elaboración de las características radiológicas que se debían valorar en la lectura de las radiografías practicadas, participaron, además del médico de vuelo de la Unidad, el médico especialista en Radiología citado y otro de los autores, especialista en Reumatología. Las variables consideradas en cada radiografia analizada presentaban respuestas dicotómicas, de modo que la valoración de las radiografías se anotó una vez por cada uno de los autores anteriormente citados, tres en total. Cada explorador desconocía la opinión de los otros dos. De este modo, en caso de opiniones divergentes siempre habría dos opiniones iguales. Este resultado es el tenido en cuenta para responder a los objetivos del presente estudio.

Tabla 1. Principales cuestiones cumplimentadas por los pilotos participantes en el estudio.

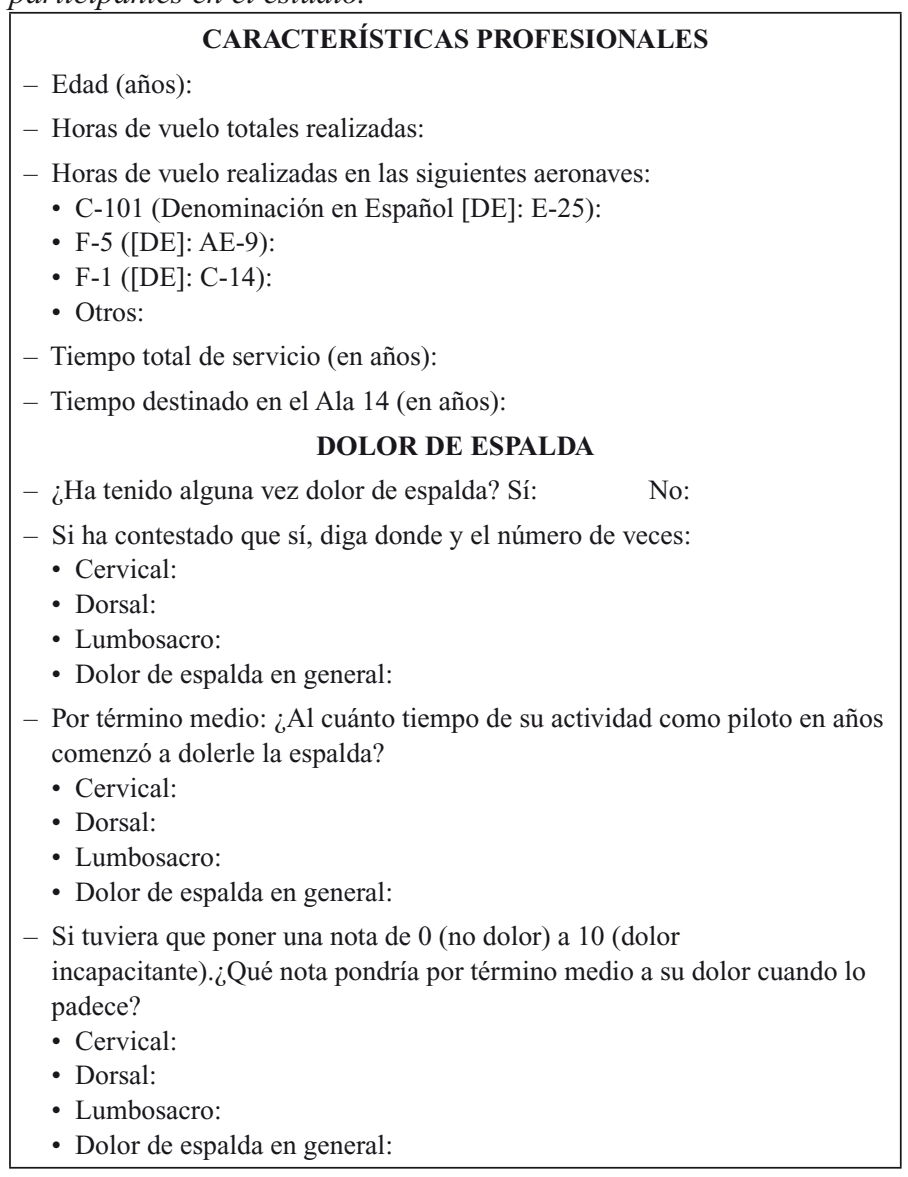

Las variables consideradas fueron: en la región cervical: perdida de lordosis, escoliosis, rotaciones, pérdida de altura del disco intervertebral, esclerosis subcondral, uncoartrosis, artrosis interapofisarias, osteofitos, hernias de Schmorl, irregularidades subcondrales, gas en disco, pérdida de altura del cuerpo vertebral, calcificaciones en disco, anomalías morfológicas, alteraciones de las apófisis espinosas, alteraciones de los pedículos y alteraciones de las partes blandas. En la columna dorsal y en el segmento lumbosacro se valoraron similares variables, si bien adaptadas a las características de cada segmento de la columna vertebral. Todas las variables referidas y su valoración se exponen en las tablas 5 (A y B), 6 (A y B) y 7 (A y B).

La movilidad de la columna vertebral de forma activa fue medida por el médico especialista en Reumatología, con amplia experiencia, el cual anotó las siguientes medidas: flexión de la columna cervical (CC), extensión de la $\mathrm{CC}$, lateralización derecha de la $\mathrm{CC}$, lateralización izquierda de la $\mathrm{CC}$, rotación derecha de la $\mathrm{CC}$, rotación izquierda de la CC, extensión de la columna lumbar (CL), lateralización izquierda de la CL, lateralización derecha de la CL, rotación derecha de la CL, rotación izquierda de la CL; todas las medidas referidas están expresadas en grados y la distancia dedos suelo en centímetros. El mismo especialista realizó las siguientes exploraciones: maniobra de compresión cervical, maniobra de Lasègue, exploración de las articulaciones sacroilíacas, movilidad de la cadera, marcha talones, marcha de puntillas y reflejos osteotendinosos.

El grupo control, con el que comparamos las características físicas medidas, no fue aleatorio sino elegido por el proceso de apareamiento, es decir, de características similares a los pilotos, excepto que no vuelan. Todos eran militares, con las mismas características de edad y antropométricas (tabla 2).

\section{Análisis estadístico}

Las variables cualitativas se expresan como cantidad exacta y en porcentaje y las cuantitativas como media y desviación estandar (DE). Cuando la dispersión de la variable cuantitativa era alta, también se expresaba la mediana. En las pruebas de hipótesis aplicamos, en presencia de variables cualitativas, como estadístico de contraste la Ji cuadrado (test de Pearson y si alguna frecuencia esperada en las casillas de la tabla de contingencia era menor de 5, la prueba exacta de Fisher). Igualmente, se calculó la razón de proporciones (razón de prevalencias [RP] en estudios transversales, la cual mide la proporción con que los hallazgos observados están presentes entre los pilotos y el grupo control). Cuando las variables eran cuantitativas y seguían una distribución normal (aplicando el test de Shapiro Wilks) en la comparación de medias utilizamos la $t$ de Student. Si no la cumplían, aplicamos la prueba no paramétrica $U$ de Mann-Whitney. El nivel máximo de error

Tabla 2. Principales características de los pilotos y del grupo control.

\begin{tabular}{|lccc|}
\hline & $\begin{array}{c}\text { Pilotos (n=30) } \\
\text { Media (DE) }\end{array}$ & $\begin{array}{c}\text { Grupo control (n=16) } \\
\text { Media (DE) }\end{array}$ & p \\
\hline Edad (años) & $31,9(5,9)$ & $32,3(3,4)$ & $\mathrm{ns}$ \\
Talla (cm) & $174,3(4,4)$ & $175,9(4,1)$ & $\mathrm{ns}$ \\
Peso (kg) & $74,3(7,1)$ & $75,5(7,5)$ & $\mathrm{ns}$ \\
IMC (kg/m2) & $24,4(2,1)$ & $24,4(1,9)$ & $\mathrm{ns}$ \\
\hline
\end{tabular}

n: número de sujetos. DE: desviación estándar. ns: no significativo. IMC: índice de masa corporal. 
alfa se fijó en igual o menos del 5\%. El análisis de los datos se ha realizado con el software SPSS (SPSS para Windows, 10.0, 1999, SPSS Inc. Chicago, IL).

\section{RESULTADOS}

Un $80 \%$ de los pilotos que cumplimentaron el cuestionario (24 de 30), refirieron padecer en alguna ocasión dolor de espalda. $\mathrm{La}$ distribución de la localización del mismo es como sigue: 20 pilotos manifestaron dolor en la columna cervical (mediana $=3,5$ veces), nueve en la columna dorsal (mediana $=3$ veces), 13 en la columna lumbar (mediana $=4$ veces) y ocho dolor de espalda global (mediana $=3$ veces). El tiempo medio de servicio activo fue de 12,6 años (mediana=11 años) y el tiempo medio destinado en el Ala 14, 4,7 años (mediana=3 años). Desde que comenzaron su actividad aeronáutica, el tiempo medio de aparición del dolor fue: segmento cervical, 6,5 años (mediana=6 años), segmento dorsal, 5,6 años (mediana $=4$ años), segmento lumbosacro, 7,1 años (mediana $=6$ años) y dolor de espalda en general, 5,8 años (mediana $=5$ años).

La experiencia en cuanto a vuelo en diferentes aeronaves se puede observar en la tabla 3 . Se trata pues, en promedio, de pilotos experimentados.

La tabla 4 refleja la movilidad de los diferentes segmentos vertebrales medidos y su comparación con el grupo control. Excepto

Tabla 3. Experiencia de vuelo en horas de los pilotos participantes.

\begin{tabular}{|lcc|}
\hline & Media $(\mathrm{DE})$ & Mediana \\
\hline Horas totales $(\mathrm{n}=30)$ & $1736,8(989,0)$ & 1750 \\
Horas en C-101 $(\mathrm{E}-25)^{*}(\mathrm{n}=29)$ & $506,5(588,3)$ & 153 \\
Horas en F-5 $(\mathrm{AE}-9)^{*}(\mathrm{n}=30)$ & $288,5(429,7$ & 107,5 \\
Horas en F-1 $(\mathrm{C}-14)^{*}(\mathrm{n}=28)$ & $803,9(734,9)$ & 638,5 \\
Otras aeronaves $(\mathrm{n}=20)$ & $239,3(434,2)$ & 100 \\
\hline
\end{tabular}

DE: desviación estándar. n: número de sujetos. *Denominación española de los tipos de aeronaves.

Tabla 4. Movilidad de la columna vertebral en los pilotos y su comparación con el grupo control.

\begin{tabular}{|lccc|}
\hline & $\begin{array}{c}\text { Pilotos (n=30) } \\
\text { Media (DE) }\end{array}$ & $\begin{array}{c}\text { Grupo control } \\
(\mathbf{n = 1 6}) \\
\text { Media (DE) }\end{array}$ & p \\
\hline Flexión columna cervical (CC) & $84,3(9,0)$ & $90(0)$ & 0,01 \\
Extensión CC & $53,8(9,7)$ & $61,9(7,5)$ & 0,006 \\
Lateralización derecha CC & $42,3(5,4)$ & $45(0)$ & 0,04 \\
Lateralización izquierda CC & $41,8(5,8)$ & $45(0)$ & 0,03 \\
Rotación derecha CC & $69,8(9,4)$ & $75(0)$ & 0,02 \\
Rotación izquierda CC & $68,6(10,8)$ & $75(0)$ & 0,01 \\
Distancia dedos suelo* & $3,9(6,0)$ & $2,7(4,3)$ & $n s$ \\
Extensión columna lumbar (CL) & $27,4(8,8)$ & $30,9(3,9)$ & 0,01 \\
Lateralización derecha CL & $35,2(4,2)$ & $37,0(1,9)$ & 0,048 \\
Lateralización izquierda CL & $35,2(4,2)$ & $37,0(1,9)$ & 0,048 \\
Rotación derecha CL & $42,8(5,0)$ & $45,3(2,8)$ & 0,006 \\
Rotación izquierda CL & $42,8(5,0)$ & $45,3(2,8)$ & 0,006 \\
\hline
\end{tabular}

n: número de sujetos. DE: desviación estándar. ns: no significativo. * Medida en centímetros. El resto de variables en grados. la distancia dedos suelo, el resto de variables presenta diferencias estadísticamente significativas, correspondiendo a los pilotos una menor movilidad.

En las tablas 5 (A y B), 6 (A y B) y 7 (A y B) podemos apreciar el nivel de significación alcanzado al comparar las variables observadas en las radiografías realizadas entre los pilotos y el grupo control, así como la RP. Se observa que las principales diferencias se encuentran en la columna cervical, apreciándose una RP más elevada en los pilotos de uncoartrosis $(\mathrm{RP}=2,4)$, artrosis interapofisarias $(\mathrm{RP}=2,82)$, osteofitos $(\mathrm{RP}=4,26)$ y pérdida de altura del cuerpo vertebral $(\mathrm{RP}=11,29)$, mientras que en la columna dorsal aparecieron más irregularidades subcondrales $(\mathrm{RP}=2,82)$, no encontrando diferencias significativas entre los pilotos y el grupo control en el segmento lumbosacro.

Cuando comparamos todas las variables descritas en las tablas 5, 6 y 7 entre los pilotos que refirieron padecer dolor de espalda $(n=24)$, frente a los que no $(n=6)$, no hallamos diferencias significativas en ninguna de las variables expuestas, es decir no encontramos asociación entre el dolor percibido y las alteraciones radiológicas observadas.

Tabla 5A. Número y porcentaje de pilotos y grupo control que presentan la variable medida en la columna cervical, grado de significación y razón de prevalencias.

\begin{tabular}{|lcccc|}
\hline & $\begin{array}{c}\text { Pilotos } \\
\text { Número } \\
\mathbf{( \% )}\end{array}$ & $\begin{array}{c}\text { Grupo } \\
\text { control } \\
\text { Número } \\
\mathbf{( \% )}\end{array}$ & $\mathbf{p}$ & $\begin{array}{c}\text { Razón de } \\
\text { Prevalencia }\end{array}$ \\
\hline Pérdida de lordosis & $15(50)$ & $10(62,5)$ & $\mathrm{ns}$ & 0,8 \\
Escoliosis & 0 & 0 & $\ldots$ & $\ldots$ \\
Rotaciones & 0 & 0 & $\ldots$ & $\ldots$ \\
PAD & $6(20)$ & 0 & $\mathrm{~ns}(0,08)$ & 5,9 \\
Esclerosis subcondral & $9(30)$ & $1(6,3)$ & $\mathrm{ns}(0,1)$ & 4,76 \\
Uncoartrosis & $18(60)$ & $4(25)$ & 0,02 & 2,4 \\
Artrosis interapofisarias & $16(53,3)$ & $3(18,8)$ & 0,02 & 2,82 \\
Osteofitos & $16(53,3)$ & $2(12,5)$ & 0,007 & 4,26 \\
Hernias de Schmorl & 0 & 0 & $\ldots$ & $\ldots$ \\
\hline
\end{tabular}

ns: no significativo. PAD: pérdida de altura del disco intervertebral.

Tabla 5B. Número y porcentaje de pilotos y grupo control que presentan la variable medida en la columna cervical, grado de significación y razón de prevalencias.

\begin{tabular}{|lcccc|}
\hline & $\begin{array}{c}\text { Pilotos } \\
\text { Número } \\
(\%)\end{array}$ & $\begin{array}{c}\text { Grupo } \\
\text { control } \\
\text { Número } \\
\mathbf{( \% )}\end{array}$ & $\mathbf{p}$ & $\begin{array}{c}\text { Razón de } \\
\text { Prevalencia }\end{array}$ \\
\hline Irregularidades subcondrales & $4(13,3)$ & $2(12,5)$ & $\mathrm{ns}$ & 1,07 \\
Gas en disco & 0 & 0 & $\ldots$ & $\ldots$ \\
PAC & $11(36,7)$ & 0 & 0,008 & 11,29 \\
Calcificaciones en disco & $2(6,7)$ & 0 & $\mathrm{~ns}$ & $\ldots$ \\
Anomalías morfológicas & 0 & 0 & $\ldots$ & $\ldots$ \\
Alt. apófisis espinosas & 0 & 0 & $\ldots$ & $\ldots$ \\
Alt. Pedículos & 0 & 0 & $\ldots$ & $\ldots$ \\
Alt. partes blandas & 0 & 0 & $\ldots$ & $\ldots$ \\
\hline
\end{tabular}

ns: no significativo. PAC: pérdida de altura del cuerpo vertebral. 
Tabla 6A. Número y porcentaje de pilotos y grupo control que presentan la variable medida en la columna dorsal, grado de significación y razón de prevalencias.

\begin{tabular}{|lcccc|}
\hline & $\begin{array}{c}\text { Pilotos } \\
\text { Número } \\
\mathbf{( \% )}\end{array}$ & $\begin{array}{c}\text { Grupo } \\
\text { control } \\
\text { Número } \\
\mathbf{( \% )}\end{array}$ & $\mathbf{p}$ & $\begin{array}{c}\text { Razón de } \\
\text { Prevalencia }\end{array}$ \\
\hline Escoliosis & $11(36,7)$ & $3(18,8)$ & $\mathrm{ns}$ & 1,96 \\
Cifosis & 0 & 0 & $\ldots$ & $\ldots$ \\
Rotaciones & $4(13,3)$ & $2(12,5)$ & $\mathrm{ns}$ & 1,07 \\
PAD & $2(6,7)$ & $1(6,3)$ & $\mathrm{ns}$ & 1,07 \\
Esclerosis subcondral & $7(23,3)$ & $3(18,8)$ & $\mathrm{ns}$ & 1,24 \\
Artrosis interapofisarias & 0 & 0 & $\ldots$ & $\ldots$ \\
Osteofitos & $11(36,7)$ & $2(12,5)$ & $\mathrm{ns}(0,1)$ & 2,96 \\
Hernias de Schmorl & $2(6,7)$ & 0 & $\mathrm{~ns}$ & $\ldots$ \\
Irregularidades subcondrales & $16(53,3)$ & $3(18,8)$ & 0,02 & 2,82 \\
\hline
\end{tabular}

ns: no significativo. PAD: pérdida de altura del disco intervertebral.

Tabla 6B. Número y porcentaje de pilotos y grupo control que presentan la variable medida en la columna dorsal, grado de significación y razón de prevalencias.

\begin{tabular}{|lcccc|}
\hline & $\begin{array}{c}\text { Pilotos } \\
\text { Número } \\
(\%)\end{array}$ & $\begin{array}{c}\text { Grupo } \\
\text { control } \\
\text { Número } \\
(\mathbf{\%})\end{array}$ & $\mathbf{p}$ & $\begin{array}{c}\text { Razón de } \\
\text { Prevalencia }\end{array}$ \\
\hline Gas en disco & 0 & 0 & $\ldots$ & $\ldots$ \\
PAC & $7(23,3)$ & $2(12,5)$ & $\mathrm{ns}$ & 1,87 \\
Calcificaciones en disco & 0 & $1(6,3)$ & $\mathrm{ns}$ & $\ldots$ \\
Anomalías morfológicas & 0 & 0 & $\ldots$ & $\ldots$ \\
Alt. apófisis espinosas & 0 & 0 & $\ldots$ & $\ldots$ \\
Alt. costo vertebrales & $1(3,3)$ & 0 & $\mathrm{~ns}$ & $\ldots$ \\
Alt. pedículos & 0 & 0 & $\ldots$ & $\ldots$ \\
Alt. partes blandas & 0 & 0 & $\ldots$ & $\ldots$ \\
\hline
\end{tabular}

PAC: pérdida de altura del cuerpo vertebral. ns: no significativo.

En todos los casos (pilotos y grupo control) la maniobra de compresión cervical fue indiferente, el signo de Lasègue estaba ausente, las maniobras de exploración de las articulaciones sacroilíacas resultaron negativas y la movilidad de la cadera fue completa. La marcha de talones y puntillas fue normal y los reflejos osteotendinosos estaban presentes. Características todas ellas normales.

Cuando aparece el dolor de espalda se automedica el 7,4\%, utilizando en ello antiinflamatorios no esteroideos y relajantes musculares. El 69,2\% ha consultado con su médico o traumatólogo, precisando tratamiento rehabilitador un $26,9 \%$. Tanto el dolor de origen cervical como lumbar son fundamentalmente fijos (el 61,9\% de origen cervical y el $75 \%$ de origen lumbar); es irradiado en el resto. Manifestaron volar con dolor de espalda el 65,4\% de los pilotos, y el 72,7\% refirió que la misión que debían realizar no se afectó por el dolor. Un $48 \%$ refiere que el dolor aparece al concluir el vuelo, asociando más frecuentemente las misiones de combate con los dolores cervical y lumbar (41,2\% para cada lugar de localización). Un $11,8 \%$ lo asocia al dolor de espalda en general y el resto $(5,8 \%)$, a otras misiones y dolor lumbar. Estos datos indican la importancia que el dolor de espalda representa para el piloto de combate, como ya ha sido citado ${ }^{1}$.
Tabla 7A. Número y porcentaje de pilotos y grupo control que presentan la variable medida en el segmento lumbosacro, grado de significación y razón de prevalencias.

\begin{tabular}{|lcccc|}
\hline & $\begin{array}{c}\text { Pilotos } \\
\text { Número } \\
(\%)\end{array}$ & $\begin{array}{c}\text { Grupo } \\
\text { control } \\
\text { Número } \\
\mathbf{( \% )}\end{array}$ & $\mathbf{p}$ & $\begin{array}{c}\text { Razón de } \\
\text { Prevalencia }\end{array}$ \\
\hline Pérdida de lordosis & $5(16,7)$ & $3(18,8)$ & $\mathrm{ns}$ & 0,89 \\
Escoliosis & $1(3,3)$ & $1(6,3)$ & $\mathrm{ns}$ & 0,53 \\
Rotaciones & $1(3,3)$ & 0 & $\mathrm{~ns}$ & $\ldots$ \\
PAD & $8(26,7)$ & $4(25)$ & $\mathrm{ns}$ & 1,07 \\
Esclerosis subcondral & $3(10)$ & $2(12,5)$ & $\mathrm{ns}$ & 0,8 \\
Artrosis interapofisarias & $5(16,7)$ & $1(6,3)$ & $\mathrm{ns}(0,6)$ & 2,7 \\
Osteofitos & $6(20)$ & $1(6,3)$ & $\mathrm{ns}(0,4)$ & 2,99 \\
Hernias de Schmorl & $1(3,3)$ & 0 & $\mathrm{~ns}$ & $\ldots$ \\
Irregularidades subcondrales & 0 & 0 & $\ldots$ & $\ldots$ \\
\hline
\end{tabular}

ns: no significativo. PAD: pérdida de altura del disco intervertebral.

Tabla 7B. Número y porcentaje de pilotos y grupo control que presentan la variable medida en el segmento lumbosacro, grado de significación y razón de prevalencias.

\begin{tabular}{|lcclc|}
\hline & $\begin{array}{c}\text { Pilotos } \\
\text { Número } \\
(\mathbf{\%})\end{array}$ & $\begin{array}{c}\text { Grupo } \\
\text { control } \\
\text { Número } \\
\mathbf{( \% )}\end{array}$ & $\mathbf{p}$ & $\begin{array}{c}\text { Razón de } \\
\text { Prevalencia }\end{array}$ \\
\hline Gas en disco & 0 & 0 & $\ldots$ & $\ldots$ \\
PAC & $2(6,7)$ & $2(12,5)$ & $\mathrm{ns}$ & 0,53 \\
Calcificaciones en disco & 0 & 0 & $\ldots$ & $\ldots$ \\
Anomalías lumbosacras & $3(10)$ & $1(6,3)$ & $\ldots$ & 1,6 \\
Anomalías morfológicas & $3(10)$ & $2(12,5)$ & $\mathrm{ns}$ & 0,8 \\
Listesis & $3(10)$ & $1(6,3)$ & $\mathrm{ns}$ & 1,6 \\
Alt. apófisis espinosas & $3(10)$ & $3(18,8)$ & $\mathrm{ns}$ & 0,53 \\
Alt. pedículos & 0 & 0 & $\ldots$ & $\ldots$ \\
Alt. partes blandas & 0 & 0 & $\ldots$ & $\ldots$ \\
\hline
\end{tabular}

PAC: pérdida de altura del cuerpo vertebral. ns: no significativo.

\section{DISCUSIÓN}

En nuestro estudio hemos encontrado que aparecen lesiones radiológicas significativas en pilotos de combate comparados con un grupo control de similares características. Hallazgos observados principalmente en la columna cervical (presencia de uncoartrosis, artrosis interapofisarias, osteofitos, pérdida de altura del cuerpo vertebral y con tendencia a la significación estadística, la pérdida de altura del disco intervertebral). En la columna dorsal solo se ha encontrado significación estadística en la presencia de irregularidades subcondrales y ninguna diferencia en el segmento lumbosacro. Alteraciones radiológicas que corresponden a degeneración del cartílago (artrosis) en una población con una edad promedio de 31,9 años, cambios degenerativos también observados por Landau et al, si bien estos autores utilizaron como medio diagnóstico imágenes de resonancia magnética ${ }^{3}$. Aunque los textos clásicos de reumatología ${ }^{4,5,6}$ no hacen referencia a la prevalencia del dolor de espalda en edades jóvenes, sí se sabe que la prevalencia del dolor cervical es alta $^{7}$, observándose que, aproximadamente, el 90\% de las personas 
mayores de 40 años presentan alteraciones radiológicas de artrosis, si bien solo un $30 \%$ presenta sintomatología ${ }^{8}$. Son conocidos los factores de riesgo que influyen en la aparición de la artrosis como la edad, el sexo, la raza, predisposición genética, obesidad, stress mecánico, traumatismos articulares, anomalías congénitas del desarrollo óseo y articular, artropatías inflamatorias y enfermedades metabólicas y endocrinas. En nuestra muestra (pilotos y grupo control), no hemos hallado en la columna cervical escoliosis, rotaciones, hernias de Schmorl, presencia de gas en el disco, anomalías morfológicas, alteraciones de las apófisis espinosas, alteraciones en los pedículos ni de partes blandas. Tampoco hemos encontrado en la columna dorsal, cifosis, artrosis interapofisarias, gas en el disco, anomalías morfológicas, alteraciones en las apófisis espinosas, alteraciones en los pedículos ni de partes blandas. En el segmento lumbosacro no hemos observado irregularidades subcondrales, gas en el disco intervertebral, calcificaciones en el disco, alteraciones en los pedículos ni anomalías en partes blandas. Es decir, nos hallamos ante una población de raza blanca, mismo sexo, similar edad, no obesa, sin anomalías congénitas de desarrollo óseo y articular, sin artropatías inflamatorias ni enfermedades metabólicas ni endocrinas. Parece que solo diferencia a ambos grupos (pilotos y grupo control) el stress mecánico a que está sometido el esqueleto axial del piloto debido a las aceleraciones $+\mathrm{Gz}$, de acuerdo con $D r e w^{9}$ y Hämäläinen et $a l^{10}$.

Por otro lado, las alteraciones radiológicas degenerativas aparecen antes en el segmento cervical y en el lumbar, segmentos de mayor movilidad. Sin embargo, las diferencias encontradas entre los dos grupos de nuestro estudio están localizadas fundamentalmente en el segmento cervical, no así en el segmento lumbosacro. A ello quizá contribuya el que, una vez en la aeronave, la columna cervical no solo mantiene la movilidad, sino que tiene que soportar un peso añadido como el casco, además del efecto propio de las aceleraciones. De hecho, se han detectado en esta región alteraciones de la función muscular, sugiriendo algunos autores una adaptación a la exposición al vuelo ${ }^{1}$ y fatiga muscular tras realizar maniobras de combate ${ }^{11}$. Una característica en la alteración del cartílago (evolución de la artrosis) es que mantiene una secuencia ordenada en la aparición de las manifestaciones radiológicas: primero se altera la pérdida de altura del disco intervertebral, seguido de la aparición de uncoartrosis, artrosis interapofisarias y en último lugar, esclerosis subcondral y osteofitos, ambos en el mismo nivel. En nuestro estudio parece que se saltaría el primer estadio para manifestarse los siguientes, correspondiendo las frecuencias más elevadas a la presencia de uncoartrosis $(60 \%)$ y artrosis interapofisarias y osteofitos (ambos con un 53\%). En cuanto a la movilidad en la columna vertebral del piloto, observamos que es menor en este grupo tal como se muestra en la tabla 4. Aunque significativas y poco relevantes, pueden representar la consecuencia del stress a que está sometida la columna vertebral, como ya se ha observado ${ }^{10}$. Así pues, contestamos al primer objetivo de nuestro estudio: sí aparecen lesiones localizadas en el segmento cervical, detectadas por radiología simple, en la muestra de pilotos participantes en nuestro estudio y al tercero: es menor la movilidad de la espalda en el piloto de combate que en el grupo comparado.

Por otro lado, es sabido que existe una escasa correlación clínico-radiológica, de modo que la manifestación clínica principal de la artrosis, el dolor, puede tener escasa representación radiológica, y al revés, alteraciones radiológicas importantes pueden ser asintomáti- cas. Los datos de nuestro estudio están de acuerdo con el enunciado anterior, ya que no hemos encontrado diferencias entre los pilotos que declararon percibir dolor de espalda y los que no. Es decir, no hallamos relación entre el dolor de espalda percibido por los pilotos y las alteraciones radiológicas observadas, con edades y experiencia aeronáutica similares.

Una vez establecidas las alteraciones radiológicas, se ha demostrado que éstas predisponen al desarrollo de la clínica en las articulaciones afectadas ${ }^{8}$ y aunque la cirugía de columna ha demostrado permitir volar a pilotos de combate españoles ${ }^{12}$, sería deseable no llegar a esta modalidad terapéutica. Por tanto, con los datos observados en nuestro estudio surgen nuevas preguntas ¿Cuándo comienza la alteración del segmento cervical en el piloto? ¿Puede prevenirse? ¿Puede prevenirse el tratamiento quirúrgico? ¿Puede disminuir el dolor percibido con otros cuidados, además de la práctica de ejercicio físico adecuado como ya ha sido descrito? $?^{2,13,14}$

Aparte del avance que pueda surgir en las condiciones ergonómicas de la cabina de las aeronaves y reducir así la fuerza ejercida sobre el segmento cervical, sí se debe aplicar un especial cuidado a la espalda del piloto de combate desde el comienzo de su vida profesional. En este sentido la mejor medida fisioterapéutica y profiláctica es una óptima preparación física ${ }^{15}$, con el fortalecimiento de los músculos paravertebrales, permitiendo una mejor distribución de las fuerzas soportadas. De hecho, se aconseja una modalidad de ejercicio físico específica para tolerar mejor las aceleraciones ${ }^{16}$. Una vez aparecida la lesión, el cuidado de la misma no difiere de las medidas habituales utilizadas en la población general.

No tenemos constancia de ningún estudio de características similares en pilotos de combate realizado en España con el que podamos comparar nuestros resultados, por lo que nuestro estudio podría ser el primero, aunque sí los hay que evalúan el dolor en pilotos de helicóptero ${ }^{17,18,19}$.

Una de las limitaciones de nuestro estudio, es que no se trata de un diseño caso-control estricto, ya que se necesita al menos igual número de controles que de casos, y ello no ha sido posible en nuestra población, sin embargo, el grupo de comparación presenta un preciso emparejamiento con el promedio de casos, incluyendo muy parecida extracción, por lo que podemos considerar válidos nuestros resultados, teniendo en cuenta esta limitación. Otra limitación es el pequeño número de participantes, pero esto es inherente a este tipo de estudios, en el que la población de estudio es, en sí, escasa.

Como resumen, diremos que aparecen lesiones precoces en la columna cervical del piloto de combate, detectadas a los 12,6 años de tiempo medio de servicio activo y que el dolor de espalda es un problema de magnitud importante (el 69,2\% ha consultado con su médico o traumatólogo, han precisado tratamiento rehabilitador un $26,9 \%$, un $65,4 \%$ ha volado con dolor de espalda y un $48 \%$ refiere que el dolor aparece al concluir el vuelo), el cual merece una especial atención. Por tanto, dentro del cuidado de la espalda debe divulgarse la práctica de ejercicio físico adecuado como la mejor medida que disponemos en la actualidad y su práctica debe ser imprescindible desde el comienzo de la actividad como piloto de combate. No obstante, podrían contemplarse otras estrategias de prevención ${ }^{20} \mathrm{de}$ índole fisioterapéutica y rehabilitadora como manipulación vertebral o terapias manuales en la columna vertebral, las cuales pueden obtener una respuesta favorable en el piloto de combate como se ha descrito recientemente ${ }^{21}$. En este sentido, ya se está realizando esta modalidad terapéutica en España con efectos alentadores. 
Los resultados de nuestro estudio aconsejan un seguimiento por imagen, fundamentalmente del segmento cervical, del piloto de combate que bien pudiera comenzar desde la estancia del futuro piloto en la Academia General del Aire, si bien la periodicidad y la modalidad radiológica seleccionada, no ha sido objetivo del presente estudio.

\section{CONCLUSIONES}

Los pilotos de combate de nuestro estudio presentan una mayor rigidez en la columna vertebral, asi como alteraciones radiológicas precoces, principalmente en la región cervical, comparados con un GC similar.

\section{BIBLIOGRAFÍA}

1. Lecompte J, Maisetti O, Guillaume A, Skalli W, Portero P. Neck strength and EMG activity in fighter pilots with episodic neck pain. Aviat Space Environ Med. 2008; 79: 947-52.

2. Carbayo JA. Percepción del dolor de espalda en pilotos de combate y su relación con la práctica de ejercicio físico. Med Mil (Esp). 1998; 54: 199-202.

3. Landau DA, Chapnick L, Yoffe N, Azaria B, Goldstein L, Atar E. Cervical and lumbar MRI findings in aviators as a function of aircraft type. Aviat Space Environ Med. 2006; 77: 1158-61.

4. Kelley WN. Testbook of Rheumatology. Philadelphia: Saunders, 1998.

5. Klippel JH, Dieppe PA. Rheumatology. Colchester (UK): Mosby, 1994.

6. Sociedad Española de Reumatología. Manual de enfermedades reumáticas. Madrid: Mosby-Doyma, 1996.

7. Haldeman S, Carroll L, Cassidy JD, Schubert J, Nygren A. The Bone and Joint Decade 2000-2010 Task Force on Neck Pain and its Associated Disorders: executive summary. Spine. 2008; 33 (4 Suppl): S5-7.

8. Hernández IR, Castillo-Ojugas A. Artrosis periférica. Medicine (Madr) 1988; 975-982.
9. Drew WE Sr. Spinal symptoms in aviators and their relationship to G-exposure and aircraft skating angle. Aviat Space Environ Med. 2000; 71: 22-30.

10. Hämäläinen O, Vanharanta H, Hupli, M, Karthu M, Kuronen P, Kinnunen H. Spinal shrinkage due to $+\mathrm{Gz}$ forces. Aviat Space Environ Med. 1996; 67: 659-61.

11. Oksa J, Hämäläinen O, Rissanen S, Salminen S, Kuronen P. Muscle fatigue caused by repeated aerial combat maneuvering exercises. Aviat Space Environ Med. 1999; 70: 556-60.

12. Vallejo P, Esteban B, López-López JA, Ríos-Tejada F, Bárcena A, Álvarez-Sala F, et al. Surgical correction of disc pathology in fighter pilots: a review of 14 cases. Aviat Space Environ Med. 2007; 78: 784-8.

13. Kemppainen $P$, Hämäläinen $O$, Könönen $M$. Different effects of physical exercise on cold pain sensitivity in fighter pilots with and without the history of acute inflight neck pain attacks. Med Sci Sports Exerc. 1998; 30: 577-82.

14. Carbayo JA, Jiménez-Prada L, Peyró R, Alonso C, Velasco C, Hernández A. Influence of Physical Exercise in the Perception of Back Pain in Spanish Fighter Pilots. Symposium NATO. 2005; Prague (Czech Republic).

15. Moreno García AC. Lesiones de la Columna Cervical en pilotos sometidos a altas aceleraciones. Medicina aeroespacial y ambiental 1994; 1: 30-33.

16. Alonso C. Ejercicios físicos para aumentar la tolerancia a las aceleraciones. Revista de Aeronáutica y Astronáutica 1986; agosto: 879-885.

17. Velasco C, Alonso C, Salinas JC, Rios F, Cantón JJ, Delgado JM, et al. Dolor de espalda en pilotos de helicóptero españoles. Sanid mil. 1990; 46: 295-6.

18. Vallejo MP, López JA, Ríos F, Jiménez R, Sierra I, García Mora L, et al. Estudio etiopatogénico de la lumbalgia de los pilotos de helicóptero. Med Aeroesp Ambient. 1999; 6: 267-75

19. López-López JA, Vallejo P, Ríos-Tejada F, Jiménez R, Sierra I, García-Mora L. Determination of lumbar muscular activity in helicopter pilots: a new approach. Aviat Space Environ Med. 2001; 72: 38-43.

20. Jones JA, Hart SF, Baskin DS, Effenhauser R, Jonson SL, Novas MA, et al. Human and behavioral factors contributing to spine-based neurological cockpit injuries in pilots of high-performance aircraft: recommendations for management and prevention. Mil Med. 2000; 165: 6-12.

21. Green BN, Dunn AS, Pearce SM, Johnson CD. Conservative management of uncomplicated mechanical neck pain in a military aviator. J Can Chiropr Assoc. 2010; 54: 92-9. 